\title{
Throughput Analysis of ALOHA with Cooperative Diversity
}

\author{
M. Sarper Gokturk, Ozgur Ercetin, and Ozgur Gurbuz
}

\begin{abstract}
Cooperative transmissions emulate multi-antenna systems and can improve the quality of signal reception. In this paper, we propose and analyze a cross layer random access scheme, C-ALOHA, that enables cooperative transmissions in the context of ALOHA system. Our analysis shows that over a

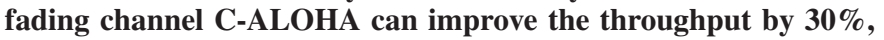
as compared to standard ALOHA protocol.
\end{abstract}

Index Terms-Wireless networks, random access schemes, cooperative communications.

\section{INTRODUCTION}

$\mathbf{C}$ OOPERATIVE communications is one of the promising techniques that can enhance the performance of wireless networks. Appropriate cross-layer medium access strategies are required to exploit the physical layer advantages provided by cooperative communications [1]. The most significant difference of a cooperative medium access protocol from a noncooperative protocol is that while conventional protocols are avoiding collisions, a cooperative protocol requires intentional collisions to boost the packet success probability.

In a wireless network, transmission errors occur either due to packet collisions or due to channel fading/noise. Recently in [2], a practical technique is presented for differentiating collisions and channel errors. In this work, we exploit the collision/error differentiation capability presented in [2] and propose an ALOHA [3] based random access scheme, Cooperative ALOHA (C-ALOHA), which enables cooperative transmissions in case of erroneous packet receptions to provide robustness to channel errors and improve the packet success probability. In C-ALOHA, initial transmission of a packet is carried out as in the ALOHA system. We assume that the receiver recognizes whether packet transmission failure is due to a collision or channel noise, and sends immediate collision or error feedbacks, respectively. In case of an error feedback, cooperative transmissions are invoked, so that some of the nodes that have correctly overheard the transmission choose to retransmit together with the source node in the next slot. Consequently, the packet success probability is improved and the number of retransmissions is decreased significantly as compared to a non-cooperative system.

Enhancing the performance of medium access methods has been investigated extensively in the literature, mainly focusing on collision avoidance, intelligent scheduling techniques and collision resolution protocols. Cross-layer approaches that

Manuscript received February 3, 2008. The associate editor coordinating the review of this letter and approving it for publication was J. Holliday. This work was in part supported by TUBITAK Career Grant No: 105 E093 and TUBITAK Grant No:105E192.

The authors are with the Faculty of Engineering and Natural Sciences, Sabanci University, Istanbul, Turkey (e-mail: msgokturk@su.sabanciuniv.edu, \{ogurbuz, oercetin\}@sabanciuniv.edu).

Digital Object Identifier 10.1109/LCOMM.2008.080174. exploit the novel physical layer findings, such as cooperative diversity, are gaining more interest. In [4], cooperative transmissions are employed to provide diversity gain to a collision resolution protocol presented in [5]. Also, recently, medium access protocols enabling cooperation in 802.11 systems are presented in [6], [7]. To the best of our knowledge, an analysis of successful packet transmission probability for a random access method in a cooperative setting still does not exist.

The contribution of this work is that a simple cross-layer ALOHA based protocol, C-ALOHA is proposed, and analytical derivation of the successful packet transmission probability is presented. C-ALOHA enables cooperative transmission for packet retransmissions in case of erroneous receptions, and thus, provides robustness to ALOHA protocol against wireless channel impairments.

\section{Preliminaries}

We consider a wireless network of $m$ nodes, where nodes transmit over a Rayleigh fading channel to a single receiver employing a Maximal-Ratio-Combiner (MRC). Packets arrive for transmission at each node according to an independent Poisson process with overall rate of $\lambda$, i.e., with rate $\lambda / m$ at each transmitting node. Each packet is of equal size and requires one time slot for transmission. All transmitters are assumed to be synchronized, and reception of each packet starts at an integer time and ends before the next integer time as assumed in the analysis of slotted ALOHA in [8]. We also assume that at the end of every slot, each node obtains immediate feedback from the receiver through an error-free channel specifying whether: 1) the slot was idle (i), 2) a transmission occurred but the packet could not be successfully decoded due to error (e),3) multiple transmissions resulted in collision $(c), 4)$ a packet was received successfully $(s)$. Errorfree feedback can be approximated by applying error control coding to the feedback packet. Furthermore, although not essential for correct protocol operation, immediate feedback is assumed to simplify the analysis.

In cooperative networks, the nodes that have correctly overheard the source transmission may cooperate with the source by retransmitting the overheard packet to the receiver, so that the receiver can be provided with multiple copies of the same signal, resulting in diversity gain. We quantify the improvement in successful packet reception probability due to cooperative transmission for varying number of cooperating nodes, when BPSK modulation is employed. The approach followed here does not depend on the modulation scheme and any modulation scheme can be used instead of BPSK. The average BER, $\bar{P}_{b}$, under Rayleigh fading for the BPSK receiver with k-branch diversity using MRC is given by [9] $\bar{P}_{b}(\bar{\gamma}, k)=$ $\frac{1}{\pi} \int_{0}^{\pi / 2}\left(1+\frac{\bar{\gamma}}{\sin ^{2} \phi}\right)^{-k} d \phi$, where $\bar{\gamma}$ refers to the average SNR 
per link, and $k$ denotes the number of cooperating nodes. Assuming uniform bit errors and no forward error correction, the success probability of a $\mu$ bit packet can be obtained as $P_{s}(k)=\left[1-\bar{P}_{b}(\bar{\gamma}, k)\right]^{\mu}$, and the probability of packet error as $P_{e}(k)=1-P_{s}(k)$. Channel SNR is assumed to be estimated at the receiver for each node-receiver link, and to remain constant for a time slot. Synchronization among transmitting nodes can be provided via techniques such as [10], and in case of imperfect synchronization, one of the methods reviewed in [11] can be employed so that full diversity gain can still be achieved.

\section{AnAlysis OF C-ALOHA}

In this section, we present the throughput analysis of the C-ALOHA protocol in detail. We assume that each node has two separate buffers, and each buffer is assumed to be of one packet size for analysis purposes. Each generated packet directly goes to buffer $B_{1}$; discarded if $B_{1}$ is full or stored otherwise. A transmitted packet is kept in $B_{1}$ until success feedback is received. All nodes in the network overhears the transmission of other nodes. In case a packet is correctly overheard, it is stored in buffer $B_{2}$, replacing the current packet in $B_{2}$. Otherwise, $B_{2}$ is emptied. Thus, $B_{2}$ always stores the correctly overheard packet in the previous slot. When the intended receiver sends an $e$ feedback, each node that correctly overheard the transmission independently transmits the packet in $B_{2}$ with probability $q_{c}$. In case of a $c$ feedback, each node independently transmits the packet in $B_{1}$ with probability $q_{r}$.

We define the state of the system with respect to the total number of backlogged packets in the network, i.e., total number of packets in $B_{1}$. The probability that a packet arrives at a node in $x$ slots is $q_{a}(x)=1-e^{x \lambda / m}$. We assume that each new arrival is not directly transmitted but backlogged. When the system is at state $n$, a successful packet reception occurs with probability $P_{n}(s)$, which can be written by conditioning on the feedback from the receiver as

$$
\begin{aligned}
P_{n}(s) & =P_{n}\left(s \mid f_{c}\right) P_{n}\left(f_{c}\right)+P_{n}\left(s \mid f_{i}\right) P_{n}\left(f_{i}\right) \\
& +P_{n}\left(s \mid f_{e}\right) P_{n}\left(f_{e}\right)+P_{n}\left(s \mid f_{s}\right) P_{n}\left(f_{s}\right),
\end{aligned}
$$

where $P_{n}\left(f_{c}\right), P_{n}\left(f_{i}\right), P_{n}\left(f_{e}\right), P_{n}\left(f_{s}\right)$ refer to the probability that $c, i, e, s$ feedbacks are received at state $n$. These probabilities are also interpreted as the probability that collision occurred in the previous slot, the previous slot was idle, erroneous or success, respectively. First, we derive $P_{n}(s)$ for ALOHA considering channel errors, and then, derive $P_{n}(s)$ for C-ALOHA.

1) $\boldsymbol{A L O H A}$ : The probability of successful transmission at state $n$ is independent of the received feedback, but only dependent on the current state. Therefore, the probability of success given the feedback, is same for each feedback, i.e., $P_{n}\left(s \mid f_{i}\right)=P_{n}\left(s \mid f_{e}\right)=P_{n}\left(s \mid f_{c}\right)=P_{n}\left(s \mid f_{s}\right)=n q_{r}(1-$ $\left.q_{r}\right)^{n-1}$.

Let $n$ be the number of backlogged nodes at an arbitrary time $t_{0}$. The system can be at any state between 0 and $n$ in the previous slot, $t_{0}-1$, depending on the number of packet arrivals during that slot. Assuming that packets arrive at the system at the end of each slot, we define the probability that $j$ arrivals occurred in $x$ slots in transition to state $n$ as $Q_{a, x}(n, j)=\left(\begin{array}{c}m-(n-j) \\ j\end{array}\right) q_{a}(x)^{j}\left(1-q_{a}(x)\right)^{m-n}$. Also, probability that $k$ nodes choose to transmit packets in the previous slot, $t_{0}-1$ is $Q_{r}(n, j, k)=\left(\begin{array}{c}n-j \\ k\end{array}\right) q_{r}^{k}\left(1-q_{r}\right)^{n-j-k}$, where $q_{r}$ is the probability that a node transmits the packet in $B_{1}$. Having quantified $Q_{a, x}$ and $Q_{r}$, we can give the probability of receiving $i, c, s, e$ feedbacks when the system is at state $n$.

$$
\begin{aligned}
& P_{n}\left(f_{i}\right)=\sum_{j=0}^{n} Q_{a, 1}(n, j) Q_{r}(n, j, 0), \\
& P_{n}\left(f_{c}\right)=1-P_{n}\left(f_{i}\right)-\sum_{j=0}^{n} Q_{a, 1}(n, j) Q_{r}(n, j, 1), \\
& P_{n}\left(f_{s}\right)=\sum_{j=0}^{n} Q_{a, 1}(n, j) Q_{r}(n, j, 1) P_{s}(1), \\
& P_{n}\left(f_{e}\right)=\sum_{j=0}^{n} Q_{a, 1}(n, j) Q_{r}(n, j, 1) P_{e}(1),
\end{aligned}
$$

where $P_{s}(1)$ and $P_{e}(1)$ refer to the success and error probabilities of a single node transmission over a noisy fading channel, respectively. The intuition behind the derivation of (2), (3) and (4) are the same in the analysis of slotted ALOHA [8]. However, (5) is derived by noting that error feedback is received when there is a single transmission but it is corrupted due to channel noise.

2) $\boldsymbol{C}-\boldsymbol{A L O H A}$ : The operation of the C-ALOHA system would be best explained by describing the response of the system to each feedback. In case of $i, c$ and $s$ feedbacks, each node transmits its packet in $B_{1}$ with probability $q_{r}$. However, in case of an $e$ feedback each node that has correctly overheard the source packet transmits the packet in $B_{2}$ together with the source node in the upcoming slot with probability $q_{c}$. Note that the first occurrence of $e$ feedback is always after a single node (direct) transmission, whereas the following occurrences can be after either a direct or a cooperative transmission. It can be easily shown that the probability of receiving an $i$ and a $c$ feedback are still given by (2), (3), respectively. The success probability given $c, i$ and $s$ feedback is $P_{n}\left(s \mid f_{c}\right)=P_{n}\left(s \mid f_{i}\right)=P_{n}\left(s \mid f_{s}\right)=n q_{r}\left(1-q_{r}\right)^{n-1}$, since after collision, idle and success events C-ALOHA operates in the same way as ALOHA.

In case of an $e$ feedback, the probability of success depends on whether or not cooperation is invoked. A packet may be received successfully after multiple erroneous trials, which may involve either cooperative or direct transmissions. We define the period between the first occurrence of the $e$ feedback and the time $s$ feedback is received as an error epoch. An error epoch may consist of multiple erroneous transmissions but can have only one successful transmission, which also designates the end of the error epoch. Let $Q_{h}(t)$ represent the probability that $t$ nodes correctly overhear the source packet, $Q_{h}(t)=$ $\left(\begin{array}{c}m \\ t\end{array}\right) P_{s}(1)^{t} P_{e}(1)^{m-t}$, and $Q_{c}(t, k)$ represent the probability that $k$ out of $t$ nodes choose to involve in cooperation, and the cooperative packet transmission is successful $Q_{c}(t, k)=$ $\left(\begin{array}{l}t \\ k\end{array}\right) q_{c}^{k}\left(1-q_{c}\right)^{t-k} P_{s}(k+1)$.

A transmission may be successful without requiring cooperation, in which case error epoch has length 1 . The 


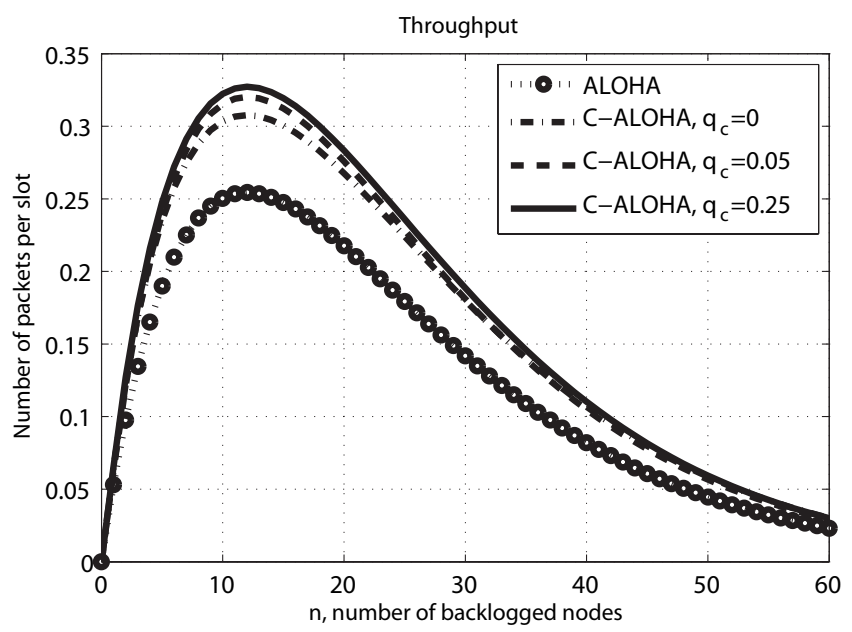

Fig. 1. Throughput for varying number of backlogged nodes.

probability of having an error epoch of length 1 is $P(l=1)=$ $\sum_{j=0}^{n} Q_{a, 1}(n, j) P_{s}(1)$. For $x \geq 2$, in order to simplify the analysis we assume that once a set of nodes chooses to cooperate with a source node, the same set of nodes continue to retransmit the packet until the end of the error epoch. Then $P(l=x)$ for $x \geq 2$ can be given

$$
\begin{aligned}
P(l=x) & =\sum_{j=0}^{n} Q_{a, x}(n, j) P_{e}(1) \sum_{t=1}^{m} Q_{h}(t) \sum_{k=1}^{t} Q_{c}(t, k) P_{e}(k+1)^{l-2} \\
& +\sum_{j=0}^{n} Q_{a, x}(n, j) P_{e}(1) \sum_{t=0}^{m} Q_{h}(t)\left(1-q_{c}\right)^{t} P_{s}(1)
\end{aligned}
$$

In the derivation of (6), we differentiate among the cases of cooperation and direct transmission employed within the epoch. The expected length of an error epoch is $E\{L\}=$ $\sum_{x=1}^{\infty} x P(l=x)$. The proportion of time the system is in error epoch is $1-P_{n}\left(f_{i}\right)-P_{n}\left(f_{c}\right)$. Since only one out of $E\{L\}$ slots is a success, by renewal-reward theory the probability of receiving $s$ feedback can be given as

$$
P_{n}\left(f_{s}\right)=\frac{1}{E\{L\}}\left[1-P_{n}\left(f_{i}\right)-P_{n}\left(f_{c}\right)\right]
$$

Furthermore, $P_{n}\left(f_{e}\right)$ is also equal to $1-P_{n}\left(f_{s}\right)-P_{n}\left(f_{c}\right)-$ $P_{n}\left(f_{i}\right)$, and $P_{n}\left(s \mid f_{e}\right)$ is

$$
P_{n}\left(s \mid f_{e}\right)=\sum_{t=1}^{m} Q_{h}(t) \sum_{k=1}^{t} Q_{c}(t, k)+\sum_{t=0}^{m} Q_{h}(t)\left(1-q_{c}\right)^{t} P_{s}(1),
$$

where the first and second terms represent the probabilities of success with cooperative transmission and direct transmission, respectively. Thus, the probability of successful transmission in C-ALOHA can be determined by (1).

\section{NumericAl RESUlts}

In this section, we compare the throughput of C-ALOHA with slotted ALOHA over a noisy fading channel. We consider independent and identically distributed (i.i.d.) node-receiver links, with Rayleigh fading, average SNR of $25 \mathrm{~dB}$, and coherence time equal to a time slot. The packet size is set as 512 bits. The throughput is calculated for varying number of backlogged nodes and different $q_{c}$ as depicted in Figure 1 . It is clearly seen that ALOHA suffers from the channel errors, and the maximum throughput is decreased dramatically to 0.255 . C-ALOHA reaches a maximum of 0.33 under the same channel conditions, slightly below the maximum throughput of slotted ALOHA in error-free channel (0.367). It is observed that the maximum throughput of slotted ALOHA can be improved by $20 \%$ by enabling error detection capability and immediate source retransmission upon error feedback. This corresponds to C-ALOHA with no cooperating nodes, i.e., $q_{c}=0$. User cooperation provides a further $10 \%$ increase in the throughput, and thus, resulting in a $30 \%$ increase in the maximum throughput of slotted ALOHA. Note that for a given channel state and network of size $m$, there is a $q_{c}$ value below which the system is underutilized, and above which the throughput can not be increased further. This is due to the fact that for a given channel state the cooperative transmission is almost always successful when the number of cooperating nodes is above a certain number, and adding additional nodes in the cooperating set does not provide any further gain.

\section{CONCLUSIONS}

In this paper, we have proposed a cross-layer random access method that incorporates cooperative transmissions into the well known ALOHA system. We have derived analytical expressions for the successful packet transmission probability for this protocol and analyzed the effect of random cooperation decision on the system performance. We have shown that by exploiting the robustness of cooperative transmissions against channel impairments, the throughput of the ALOHA system in a noisy fading channel can be improved by $30 \%$.

\section{REFERENCES}

[1] F. H. P. Fitzek and M. D. Katz, Cooperation in Wireless Networks: Principles and Applications, 1st ed. Dordrecht, The Netherlands: Springer, 2006.

[2] K. Whitehouse, A. Woo, F. Jiang, J. Polastre, and D. Culler, "Exploiting the capture effect for collision detection and recovery," in Proc. Em-NetSII, pp. 45-52, May 2005.

[3] N. Abramson, "The Aloha system-another alternative for computer communications," in Proc. AFIPS Conf., vol. 37, pp. 281-285, Montvale, NJ, 1970.

[4] R. Lin and A. R. Petropulu, "Cooperative transmission for random access wireless networks," in Proc. Asilomar Conf., vol. 2, pp. 1922-1926, Nov. 2004.

[5] M. K. Tsatsanis, R. Zhang, and S. Banerjee, "Network-assisted diversity for random access wireless networks," IEEE Trans. Signal Process., vol. 48, no. 3, pp. 702-711, Mar. 2000.

[6] P. Liu, Z. Tao, S. Narayanan, T. Korakis, and S. S. Panwar, "CoopMAC: a cooperative MAC for wireless LANs," IEEE J. Select. Areas Commun., vol. 25, no. 2, pp. 340-354, Feb. 2007.

[7] M. S. Gokturk and O. Gurbuz, "Cooperation in wireless sensor networks: design and performance analysis of a MAC protocol," in Proc. ICC'08.

[8] D. Bertsekas and R. Gallager, Data Networks, 2nd ed. Prentice Hall, 1992.

[9] A. J. Goldsmith, Wireless Communications, 1st ed. New York: Cambridge University Press, 2005.

[10] D. L. Mills, "Internet time synchronization: the network time protocol," IEEE Trans. Commun., vol. 39, no. 10, pp. 1482-1493, Oct. 1991.

[11] G. Jakllari, S. V. Krishnamurthy, M. Faloutsos, P. V. Krishnamurthy, and O. Ercetin, "A cross-layer framework for exploiting virtual MISO links in mobile ad hoc networks," IEEE Trans. Mobile Comput., vol. 6, no. 6, pp. 579-594, June 2007. 